\title{
Leading Students to Investigate Diffusion as a Model of Brine Shrimp Movement
}

\author{
Brynja R. Kohler ${ }^{\mathrm{a}, *}$, Rebecca J. Swank ${ }^{\mathrm{a}}$, James W. Haefner ${ }^{\mathrm{b}}$, \\ James A. Powella,b \\ ${ }^{a}$ Department of Mathematics and Statistics, Utah State University, Logan, UT 84322-3900, \\ USA \\ ${ }^{b}$ Department of Biology, Utah State University, Logan, UT 84322-5305, USA
}

Received: 17 December 2008 / Accepted: 16 July 2009 / Published online: 9 October 2009

(C) Society for Mathematical Biology 2009

\begin{abstract}
Integrating experimental biology laboratory exercises with mathematical modeling can be an effective tool to enhance mathematical relevance for biologists and to emphasize biological realism for mathematicians. This paper describes a lab project designed for and tested in an undergraduate biomathematics course. In the lab, students follow and track the paths of individual brine shrimp confined in shallow salt water in a Petri dish. Students investigate the question, "Is the movement well characterized as a 2-dimensional random walk?" Through open, but directed discussions, students derive the corresponding partial differential equation, gain an understanding of the solution behavior, and model brine shrimp dispersal under the experimental conditions developed in class. Students use data they collect to estimate a diffusion coefficient, and perform additional experiments of their own design tracking shrimp migration for model validation. We present our teaching philosophy, lecture notes, instructional and lab procedures, and the results of our class-tested experiments so that others can implement this exercise in their classes. Our own experience has led us to appreciate the pedagogical value of allowing students and faculty to grapple with open-ended questions, imperfect data, and the various issues of modeling biological phenomena.
\end{abstract}

Keywords Project-based learning · Undergraduate education · Brine shrimp · Artemia franciscana $\cdot$ Diffusion $\cdot$ Random walks

\section{Introduction}

Generally speaking, college mathematics teaching should evolve to better address the needs of nonmathematicians in our rapidly changing, technologically advanced, and information-abundant society. Mathematical pedagogy should train future scientists to

\footnotetext{
*Corresponding author.

E-mail address: Brynja.Kohler@usu.edu (Brynja R. Kohler).
} 
reason inductively, discern patterns in complex data, apply mathematics in novel empirical contexts, and communicate results clearly to a broad audience. Teaching must therefore address multiple aspects of cognition, and students should learn to prepare written and verbal presentations consistent with the standards and format of general scientific communication. In this paper, we describe a two-week class project designed for a biomathematics course in which students integrate experiments and models to investigate the applicability of diffusion for understanding the dispersal of brine shrimp.

The brine shrimp diffusion project provides students with the experience of dealing with the imperfections and realities of data collection, parameter estimation, and model validation, thus narrowing the gap between instruction and research practices. Our goal for this paper is to provide enough mathematical, biological, and pedagogical content so that a mathematics professor teaching applied mathematics, differential equations, or statistics (or a biology professor teaching a quantitative or modeling-oriented course) has sufficient information to comfortably undertake this investigation in his or her class.

The paper proceeds with a general introduction to our pedagogical framework and educational approaches, the course in which we have implemented the project, and our motivation for addressing diffusion and movement. A methods section describes both the mathematical background and biological basics for conducting the discussions and experiments required for the exercise. Teaching notes are interspersed throughout this paper. In the results section, model parameterization and validation results are analyzed and presented from our own experiments to help the implementation of the project. A summary of student results is also included to illustrate the achievement of learning goals associated with this project. A concluding discussion section summarizes our experiences and findings regarding best classroom practices and possible adaptations and extensions of this movement activity.

\subsection{Pedagogical framework}

One important component of any instructional design is the consideration of how students will interact with the mathematical content. Some educators believe that the central focus of teaching should be on the transfer of knowledge, while others emphasize that students need to be actively engaged in the construction of knowledge for themselves. The scheme displayed in Table 1 allows us to take both perspectives into account with definitions that describe the various cognitive activities that pertain to learning mathematics (Cangelosi, 2003). We use this scheme for organizing lesson sequences, articulating specific learning objectives, selecting appropriate teaching strategies, and evaluating the achievement of our learning goals. For example, while direct instructional strategies (such as lecture) are appropriate for accomplishing simple knowledge or algorithmic skill objectives, inquiry instructional strategies are most effective for attaining conceptual, discovery, application, and creative thinking objectives (Cangelosi, 2003). To achieve this wide array of cognitive objectives, we engage students in project-based learning.

The project-based learning (PBL) model focuses on driving questions or problems requiring core concepts of the curriculum (Krajcik et al., 1994). While addressing the central driving question, students engaged in PBL are involved in experimental design, data collection and analysis, problem solving, inquiry, and decision making that culminates in realistic products such as presentations, written reports, or physical models (Thomas, 2000; Solomon, 2003). Throughout the learning process, the role of the teacher is to introduce 
Table 1 Scheme to classify the learning levels of mathematical lesson objectives (Cangelosi, 2003)

\begin{tabular}{lll}
\hline Domain & Learning Level & $\begin{array}{l}\text { Examples of learning objectives } \\
\text { students attained through this project }\end{array}$ \\
\hline
\end{tabular}

Cognitive Construct a concept

Use inductive reasoning, distinguish between examples and nonexamples

\section{Discover a relationship}

Use inductive reasoning, discover relationships among concepts

\section{Simple knowledge}

Recall a specified response (not multistep) to a specified stimulus

Comprehension and communication Extract and interpret meaning, use the language of mathematics

\section{Algorithmic skill \\ Recall and execute a multistep procedure}

\begin{abstract}
Application
Use deductive reasoning, decide if at all mathematical content is relevant
\end{abstract}

\section{Creative thinking}

Use divergent reasoning to view mathematical content in unusual, novel ways

Affective $\begin{aligned} & \text { Appreciation } \\ & \text { Believe mathematical content has value }\end{aligned}$
Willingness to try
Choose to attempt a mathematical task

Provided examples of diffusion or random motion in nature such as movement of ions, dispersal of particles, loss of heat

Observed, explained and quantified patterns in the shrimp movement. Related the mathematical model to these observations and discovered the complexity involved in relating experiment and theory. Invented and refined experimental protocols for data collection such as how to estimate $N_{0}$

Recalled the PDE for diffusion and the correct units for the diffusion coefficient

Integrated quantitative findings and mathematical formulae in a written report of experimental results. Used the appropriate commands and made meaningful plots using MATLAB

Systematically gathered data for each experimental procedure. Estimated parameters for the arrival densities validation using linear regression

Developed a prediction for the existence of a peak in the arrival rate results based on PDE solution. Invented plausible explanations for the effects of Petri dish edges on the randomness of observed shrimp movement

Provided novel explanations for discrepancies between data and model predictions

Expressed an interest in quantifying observations made about the cute and likable shrimp

Included an analysis of the sensitivity of parameters in the lab report

relevant content before and during the lesson as well as guide and advise students rather than directly manage student work (Solomon, 2003; Hmelo-Silver, 2004). There is not one universally accepted model of PBL, but the following are defining characteristics typically found in learning activities described as project-based learning.

First, students are confronted with authentic problems that are both central to the curriculum and do not have a predetermined solution (Thomas, 2000; Solomon, 2003). This means that in PBL, projects do not serve to illustrate examples of concepts previously 
taught or to provide extra practice. Rather, students learn the central concepts of the curriculum through the project. Also, the driving questions in a PBL learning activity cannot be so constrained as to restrict students from developing their own solution methods. Students must be granted a certain degree of autonomy, as well as more choice and responsibility than is typically seen in traditional teaching methods (Thomas, 2000).

Second, PBL activities involve students in investigations. These investigations could take the form of experimental design, problem solving, decision making, or model building, with the requirement that existing knowledge is connected to newly constructed knowledge throughout the inquiry processes of the investigation (Thomas, 2000). Throughout the PBL experience, students ask and refine questions, explore ideas, make predictions, conduct experiments, analyze data, and draw conclusions while gaining new knowledge (Krajcik et al., 1994). By the end of the PBL experience, students will have generated artifacts that represent the solution process and solution based upon these investigations.

Lastly, the driving question around which a PBL experience is formed must be an authentic, real world problem (Solomon, 2003). The problem must be worthwhile, by containing important content and reflecting what actual practitioners of the given discipline might encounter, while also being feasible as a source for student learning (Krajcik et al., 1994). The success of PBL can depend largely on the chosen question, as well as on the tasks and roles given to students to carry out during the learning process. According to Erickson, when choosing problems, teachers should make sure that they are genuine problems that reflect the goals of instruction, situations that consider students' interests and experiences, appropriate content considering students' prior knowledge, and of a difficulty level that will challenge the students without discouraging them (Erickson, 1999). This last factor also leads to an additional challenge for the teacher implementing problem-based instruction. When presenting students with problems that are challenging and do not immediately lend themselves to a certain solution strategy, teachers need to be willing to let students struggle without offering suggested methods. However, teachers also need to provide students with sufficient guidance to keep them interested in the problem and focused (Erickson, 1999).

Many resources on undergraduate mathematics teaching have recently been published that support the general integration of education research findings and trends into undergraduate mathematics instruction. The reader may find some of the following resources useful in the endeavor to improve teaching. The CUPM Curriculum Guide makes recommendations for undergraduate programs and courses in mathematics and outlines the need for action due to the increasing scientific and technological demands of our society (Pollatsek et al., 2004). For ideas to get students working together, see Cooperative Learning (Rogers et al., 2001). Krantz's book How to Teach Mathematics, Second Edition, emphasizes the traditional lecture technique, but also contains informative appendices by various mathematics education researchers that address reform-oriented teaching strategies (Krantz, 1999). Some researchers have specifically studied the implementation of inquiry strategies for differential equations concepts, and one example of the results of such research is the formulation of specific types of pedagogical tools (Rassmussen and Marrongelle, 2006). The book Math\&Bio 2010 offers a variety of articles on the issues specific to successfully integrating biology and mathematics (Steen, 2005). Following up on that report, a recent special issue from the journal PRIMUS Problems Issues and 
Resources in Mathematics Undergraduate Studies on "Integrating Mathematics and Biology" contains several articles with specific examples of curricular links and teaching approaches from other institutions (Ledder, 2008).

\subsection{Introduction to the class}

The class project presented here is part of a Utah State University class for advanced undergraduate or beginning graduates entitled "Applied Mathematics in Biology" (AMB). It was originally developed as part of a program to introduce mathematical modeling elements into existing biology laboratory classes. The program aimed to counteract the negative reactions of biology students to required mathematics and statistics courses by introducing mathematical components throughout the biology curriculum. Thus, we developed modeling modules for courses from introductory freshman biology through advanced physiology and genetics courses. In addition, we developed a capstone course, AMB, for students in USU's BioMath minor as well as those wanting more exposure to the interface of mathematics and laboratory experimentation. The motivation was to confront students with the experience of doing mathematical biology in the real world context.

AMB is open to both biologists with little mathematics (often a year of calculus) and mathematicians with little or no biology experience. This openness has disadvantages and advantages, as it implies wide differences in expertise. Lecture-based instruction is particularly difficult as content background challenges only part of the class. However, it provides a natural test situation for project-based learning, and permits a rare opportunity for both sets of students to engage in a real world situation: collaborating with scientists from disparate disciplines.

The course content consists of a series of projects that integrate laboratory exercises and model development and analysis, team taught by a biologist (Haefner) and a mathematician (Powell). We have taught it to as few as 5 students and as many as 12 . The overall learning goals are: (1) developing multiple mathematical models from general biological questions (e.g., What size of prey should a fish eat?); (2) developing an experimental design appropriate to selecting among the models; (3) estimating parameters and statistically testing model predictions with the data; (4) selecting the best of several models; (5) experience in handling biological material and dealing with messy, noisy data; (6) presenting basic tools to analyze the model behaviors (e.g., nullclines, stability analysis); and (7) creating a learning environment that fosters social skills needed for integrative research. The specific topics covered were chosen to address these goals, not to cover any particular canon of knowledge. Some of the topics covered are: epidemiology games (Powell et al., 1998), fluids flowing from containers, yeast population growth, optimal foraging in fish, and spatial movement of brine shrimp.

\subsection{Why diffusion models?}

Almost all biology occurs in a spatial context; almost every process, from propagation of nerve impulses and oxygenation of tissue to the spread of forests after the last ice age, involves movement in space. At times and small enough scales this movement may seem very directed, as when ants follow pheromone trails or squirrels move seeds; at others it is seemingly random, as in the sprint and tumble of E. coli, the foraging of ladybird larvae, 
or the dispersal of pollen in the wind. Even directed movement can be apparently random if the cues which give it direction are stochastic from an observer's standpoint. Depending on the interaction between movement mechanisms, spatial heterogeneity and biological process, models must often account for the effects of movement to faithfully describe the natural world.

Among the most celebrated models in mathematical biology are those with a diffusive term, including Fisher's 1937 discussion of the spread of advantageous mutations into a population, spatial models such as Skellam's 1951 description and analysis of invasion following a population introduction, and Turing's 1952 use of diffusion models and their instabilities to provide a theory for pattern formation (as with stripe and spot patterns in the hides of animals) (Fisher, 1937; Skellam, 1951; Turing, 1952). Since these classics, there have been thousands of papers and many books written about the effect of random movement on biological dynamics. Berg's 1993 book is an excellent starting reference on the diffusion equation, which is the canonical PDE description of the random transport of populations of entities (either chemical or organismal) (Berg, 1993). A special issue of Ecology in 1994 (Miller, 1994) devoted to spatial modeling is a useful survey from an ecological perspective, and Turchin's 1998 book gives practical advice on connecting diffusion models to animal movement (Turchin, 1998).

\section{Methods}

Our primary pedagogical approach with this project is to maintain a loosely guided conversation with our students, and not to instruct them through a series of prescribed steps. Although very few have been introduced to partial differential equation (PDE) movement models, we attempt to arrive at the labs collectively as a natural means for investigating dispersal. Hence, our unit of instruction begins with the general observation that animals and plants move in space. We provide some historical background, similar to that outlined above, and initiate a discussion of patterns of animal movement. This can be sophisticated if certain types of biologists are in the class, but in any case, everyone has watched ants or ladybird beetles or seen fish swim, so there is always a common background. Through directed discussion, we lead the students to the role of randomness in searching decisions. From this, a probabilistic derivation follows naturally, and then to the continuous time system and PDEs. Details of this derivation are presented below.

Once the basic mathematical framework is available, more directed discussion gets the class to study small animals either swimming or crawling. The fundamental biological assumptions in a diffusion model (e.g., no individual interactions, no effect of external environment) are elicited from the students and considered in the context of choosing an experimental organism and protocols. We introduce the brine shrimp and their biology, and discuss experimental objectives and constraints imposed by available materials and space. Biological and experimental details are presented below. Depending on the timing of class periods, it can be profitable to have these discussions in a class period before the experiments are performed, allowing students more input into variations of the basic experimental design.

Students must revisit the mathematical model describing diffusion and discuss how essential parameters will be estimated given available protocols. Generally speaking, the easiest way to do this is by observations of individual movement trajectories and analysis 
of mean-squared displacements; details on why this works and how students can be mathematically responsible for regression are presented below. However, this is not nearly the only way to arrive at diffusion parameters. Presenting parameterization as a problem to be solved by the group is essential to student involvement, and in our experience also essential to student internalization of mathematical concepts. The issue of numbers of replicates and the role of sensitivity analysis should be discussed at this point, also; a formal sensitivity analysis is presented below to provide a context that will allow instructors to usefully guide this discussion.

The project follows the two basic steps of all mathematical biosciences: parameterization and validation/falsification. Here, we present the most common form that the brine shrimp diffusion project takes; (1) students follow individual shrimp and record positions and times to estimate a diffusion coefficient $D$, and (2) students count numbers of shrimp in regions over time for comparison with analytic predictions. All students do both activities in small groups; sizes of groups are discussed below. If preparations are limited, it is possible for some groups to do (2) before (1), but all students should do both. We generally frame the entire project as a question, "Do brine shrimp move randomly?" and present the mathematical framework for population predictions as a consequence of random motion as a "null" model which students may or may not choose to reject.

\subsection{Mathematical methods}

At a population level, what are the consequences of assuming that shrimp move completely at random? What follows is a summary of the mathematical background needed for investigating diffusive aspects of brine shrimp movement. A lattice version of random movement, leading to the diffusion model, is presented and discussed pedagogically. Lecture notes are provided for solution behavior and linear regression for finding diffusion constants (as well as many other parameters in the rest of AMB). For model validation, we develop diffusion predictions for an initial point release of shrimp, and summarize the appropriate sensitivity coefficients for parameters in the point release model to give instructors a notion of where student measurement efforts may be most fruitfully focused.

\subsubsection{Derivation of diffusion equation}

There are many ways to derive the diffusion equation. In advanced engineering and mathematics classes, a flux-based derivation is often used. A difficulty with the flux-based derivation is that it depends on a number of relatively advanced mathematical concepts for both particular steps and general understanding. For example, the notion that gradients point directly uphill and, therefore, random motion will, on average, create a flux of individuals downhill (parallel to the negative gradient) often loses students. Applying the divergence theorem, to pass from fluxes through boundaries to divergence of flux inside the area, may not be in the front of most students' minds; only rarely do biology students take multi-variable calculus. Consequently the flux-based derivation becomes one of many slick, elegant presentations that students will accept, but may not understand. We generally opt for a more constructive approach, based on random walks.

Imagine space partitioned into a grid of (small) rectangles of width $\Delta x$ and breadth $\Delta y$. Let $N(x, y, t)$ denote the number of individuals in the rectangle centered at the point $(x, y)$ at time $t$. Suppose that during each step of time, $\Delta t$, individuals in each box choose to move either left or right, either forward or backward, with no bias. We ask 
students then to predict the number of individuals at $(x, y)$ one step in the future. After some discussion, they realize that individuals will all have come from directly neighboring cells in a "+" pattern, each of those neighbors contributing on average one quarter of the individuals originally resident. Thus,

$$
\begin{aligned}
N(x, y, t+\Delta t)= & \frac{1}{4}[N(x-\Delta x, y, t)+N(x+\Delta x, y, t) \\
& +N(x, y-\Delta y, t)+N(x, y+\Delta y, t)] .
\end{aligned}
$$

It is important to connect with densities ( $P$ measured in organisms/area), so that numbers of organisms in arbitrary areas can be analyzed. For suitably small rectangles, $N \approx P \Delta x \Delta y$, with the degree of the approximation improving as the rectangles become smaller. Then (1) holds for the density function, substituting $N=P \Delta x \Delta y$ and canceling terms.

We seek an equation which holds at a single $(x, y, t)$ location. Accordingly, we expand $P$ (legitimately a smooth function, unlike $N$ ) in a Taylor series,

$$
\begin{aligned}
& P(x \pm \Delta x, y, t)=P(x, y, t) \pm \Delta x P_{x}+\frac{1}{2} \Delta x^{2} P_{x x}+\cdots, \\
& P(x, y \pm \Delta y, t)=P(x, y, t) \pm \Delta y P_{y}+\frac{1}{2} \Delta y^{2} P_{y y}+\cdots, \\
& P(x, y, t+\Delta t)=P(x, y, t) \pm \Delta t P_{t}+\cdots
\end{aligned}
$$

where we have adopted subscript notation for partial derivatives and all functions are evaluated at $(x, y, t)$. Substituting into (1) gives

$$
\begin{aligned}
& P(x, y, t)+\Delta t P_{t}+\cdots \\
& =\frac{1}{4}\left[P(x, y, t)+\Delta x P_{x}+\frac{1}{2} \Delta x^{2} P_{x x}+P(x, y, t)-\Delta x P_{x}+\frac{1}{2} \Delta x^{2} P_{x x}\right. \\
& \left.\quad+P(x, y, t)+\Delta y P_{y}+\frac{1}{2} \Delta y^{2} P_{y y}+P(x, y, t)-\Delta y P_{y}+\frac{1}{2} \Delta y^{2} P_{y y}+\cdots\right] .
\end{aligned}
$$

Students get excited canceling the obvious terms, leading to

$$
\Delta t P_{t}=\frac{1}{4}\left[\Delta x^{2} P_{x x}+\Delta y^{2} P_{y y}\right]+\cdots .
$$

If $\Delta x=\Delta y$ (that is, movement is isotropic, or step size is equal in both directions) and we take the distinguished limit $\Delta t, \Delta x, \Delta y \rightarrow 0$ while $D=\frac{\Delta x^{2}+\Delta y^{2}}{4 \Delta t}$ is held constant, we arrive at the diffusion equation

$$
P_{t}=D\left[P_{x x}+P_{y y}\right]
$$

For future reference, note that the diffusion constant can be interpreted

$$
D=\frac{\Delta x^{2}+\Delta y^{2}}{4 \Delta t}=\frac{\text { Mean Squared Distance Moved }}{4 \cdot \text { Time to Move That Far }} .
$$


This derivation, in our experience, invites student discussion and participation and is also amenable to experimentation-students can put a pile of coins in the center of a square grid and start flipping each coin (twice) and moving it left, right, up, or down as indicated. A few iterations convey the behavior of the diffusion equation.

\subsubsection{Solutions and behavior}

At this point we generally exhibit a solution for discussion,

$$
P(x, y, t)=\frac{1}{4 \pi D t} e^{-\frac{x^{2}+y^{2}}{4 D t}} .
$$

This is the fundamental solution, which is found using similarity and transform methods in PDE and applied mathematics classes. In a multivariate calculus setting, this solution is often given as an applied example in partial differentiation and the utility of higher partial derivatives (e.g., testing to see that (3) is actually a solution to the diffusion equation). In all classes, we discuss behavior of the solution, including convergence to the delta function as time tends to zero and what this means from an observational and biological perspective. We also make some connection with the statistical background. Since (3) can be viewed as the product of two normal distributions for independent $x, y$ variables,

$$
\frac{1}{4 \pi D t} e^{-\frac{x^{2}+y^{2}}{4 D t}}=\frac{1}{\sqrt{4 \pi D t}} e^{-\frac{x^{2}}{4 D t}} \frac{1}{\sqrt{4 \pi D t}} e^{-\frac{y^{2}}{4 D t}},
$$

each with variance $\sigma^{2}=2 D t$, the variance of sums of squares of these variables is additive,

$$
E\left(x^{2}+y^{2}\right)=\sigma^{2}+\sigma^{2}=4 D t .
$$

This means that the characteristic area covered by the population profile grows linearly with time, while the peak density decreases inversely with time.

\subsubsection{Regression to find diffusion constants}

Discussion of population variance following point release is important not only because it increases understanding of solution behavior, but also because this provides an avenue by which researchers estimate $D$ for real populations (Turchin, 1998). Since the expectation of squared distances increases linearly (4), it follows that if squared displacements of several individuals are averaged and observations of mean squared displacement are plotted in time they should produce a line with slope $4 D$ passing through the origin. Students must have a method to determine the most likely slope from imperfect data-a special case of linear regression. Students at many levels can benefit from a discussion of regression with general linear models, and its basis in linear algebra, in order to figure out the slope of a regression line passing through the origin. Most linear algebra books, and most linear algebra and basic statistics classes, seem to skip over this crucial piece of mathematical technology, so we present the approach in detail, following the discussion given in Lay (2003).

Generally, if a model

$$
x=a_{1} f_{1}(t)+a_{2} f_{2}(t)+\cdots+a_{m} f_{m}(t)
$$


is to be fit to data $\left\{\left(t_{j}, x_{j}\right)\right\}_{j=1}^{n}$, then the system of equations

$$
x_{j}=a_{1} f_{1}\left(t_{j}\right)+a_{2} f_{2}\left(t_{j}\right)+\cdots+a_{m} f_{m}\left(t_{j}\right)
$$

must be solved for the coefficients $a_{i}$. The system can be written in matrix form

$$
\vec{x}=\mathbf{F} \vec{a},
$$

where $\vec{x}$ is the vector of observations, $\vec{a}$ is the vector of coefficients, and $\mathbf{F}$ is called the design matrix with rows $(i)$ given as the individual model functions evaluated at $t_{i}\left(f_{j}\left(t_{i}\right)\right)$. The system is overdetermined when $n>m$, and so solutions normally only exist in the least squares sense. Following Lay (2003), the system

$$
\mathbf{F}^{\mathrm{T}} \vec{x}=\left(\mathbf{F}^{\mathrm{T}} \mathbf{F}\right) \vec{a}
$$

is the orthogonal projection of these equations onto the space spanned by the model functions; therefore,

$$
\hat{a}=\left(\mathbf{F}^{\mathrm{T}} \mathbf{F}\right)^{-1} \mathbf{F}^{\mathrm{T}} \vec{x}
$$

is the "best" solution in the least-squares sense. The matrix $\left(\mathbf{F}^{\mathrm{T}} \mathbf{F}\right)^{-1} \mathbf{F}^{\mathrm{T}}$ is called the pseudo-inverse. (Just a note for mathematicians-correlations among the model functions across $t_{i}$ appear as near collinearity of columns in the design matrix, which leads to poor conditioning in the inverse.) We generally spend a lecture and a computer lab on this subject in the AMB class since determining parameters from experimental data is probably the single most important technical proficiency required for biomathematics students. Students are encouraged to write MATLAB scripts that implement their own understanding of the algorithms needed for their analysis. Some use built-in functions in MATLAB or Excel spreadsheets, but they usually require tweaking to force a line through zero. Most mathematics students, even those with advanced linear algebra experience, have not seen the connection between projections, least squares solutions, pseudo-inverses, regression, and parameter estimation, so this content is invariably an eye-opener. We have found that students benefit from heterogeneous groups in which at least one member comfortably can write and execute simple programs.

In the case of fitting a line with intercept at the origin, the design matrix is a vector,

$$
\mathbf{F}=\left(t_{1}, t_{2}, \ldots, t_{n}\right)^{\mathrm{T}},
$$

so the slope is just

$$
\hat{a}_{1}=\frac{\left(t_{1}, t_{2}, \ldots, t_{n}\right)\left(r_{1}, r_{2}, \ldots, r_{n}\right)^{\mathrm{T}}}{\left(t_{1}, t_{2}, \ldots, t_{n}\right)\left(t_{1}, t_{2}, \ldots, t_{n}\right)^{\mathrm{T}}},
$$

where the $r_{j}$ are mean squared displacements at time $t_{j}$.

\subsubsection{Arrival predictions for validation}

An overall theme in AMB is validation/falsification of parameterized models in the arena of independent experiments. Ideally students construct the validation experiments themselves, often on the basis of situations we have discussed in class. One example involves 
release of a population and then subsequent observation to determine whether dispersal assumes a Gaussian profile, but typically this involves a lot of counting and it is difficult to count rapidly enough to capture an accurate snapshot of the spatio-temporal process. Another example is observations of arrival density in a small target area following a "point" introduction, simulating arrival of exotic or diseased populations in a conservation area at some distance from an initial introduction. This is pedagogically interesting, since it can be attached to a variety of stories (e.g., at what rate should national park managers expect arrival of deer with chronic wasting disease if the park is some distance from a point source of infection) and leads to a good discussion of pulse behavior in the diffusion equation. Will there (can there?) be a maximum in the number arriving? If so, when should it be expected and how long until it fades away? These questions can be illuminated by considering the asymptotic cases: at time zero, there should be no arrivals, and as time goes on to eternity the population should become evenly dispersed at low density (or zero density in an infinite space). Somewhere in between then must be a maximum rate of arrival (which can lead to more discussion in a PDE context, since diffusion solutions can have no internal extrema).

A little preliminary experimentation reveals that "point" release of a population is impossible. Via either discussion (beginning with the statistical nature of the diffusion solution and estimating the variance of shrimp following point release) or instructor revelation we introduce an extension of (3),

$$
P(r, t)=\frac{N_{0}}{2 \pi\left(\sigma_{0}^{2}+2 D t\right)} e^{-\frac{r^{2}}{2\left(\sigma_{0}^{2}+2 D t\right)}},
$$

where $\sigma_{0}$ characterizes the spatial extent of the initial release and $N_{0}$ is the number of individuals released. The predicted number of individuals in a counting area, $A$, would be

$$
N_{A}(t)=\int_{A} \int P(r, t) d A=\frac{N_{0}}{2 \pi} \int_{\alpha}^{\beta} \int_{L_{1}}^{L_{2}} e^{-\frac{r^{2}}{2\left(\sigma_{0}^{2}+2 D t\right)}} \frac{r}{\sigma_{0}^{2}+2 D t} d r d \theta
$$

where $A$ is an annular region contained between the angles $\alpha$ and $\beta$ with $L_{1} \leq r \leq L_{2}$. The substitution

$$
u=\frac{r^{2}}{2\left(\sigma_{0}^{2}+2 D t\right)}
$$

gives

$$
N_{A}(t)=N_{0} \frac{\beta-\alpha}{2 \pi}\left[e^{-\frac{L_{1}^{2}}{2\left(\sigma_{0}^{2}+2 D t\right)}}-e^{-\frac{L_{2}^{2}}{2\left(\sigma_{0}^{2}+2 D t\right)}}\right] .
$$

Alternatively, the prediction

$$
N_{A}(t) \approx N_{0} P(L, t)=\frac{N_{0} A}{2 \pi\left(\sigma_{0}^{2}+2 D t\right)} e^{-\frac{L^{2}}{2\left(\sigma_{0}^{2}+2 D t\right)}}
$$

is reasonable for a class that is not up to the double integration, provided the radial extent of the area $A$ is small. An interesting problem for more advanced students is to determine 
the order of accuracy of the approximation. Letting $L_{1}=L-\Delta$ and $L_{2}=L+\Delta$ in (6),

$$
\begin{aligned}
N_{A} & =N_{0} \frac{\beta-\alpha}{2 \pi}\left[e^{-\frac{(L-\Delta)^{2}}{2\left(\sigma_{0}^{2}+2 D t\right)}}-e^{-\frac{(L+\Delta)^{2}}{2\left(\sigma_{0}^{2}+2 D t\right)}}\right] \\
& =N_{0} \frac{\beta-\alpha}{2 \pi} 2 \Delta \frac{d}{d L}\left[-e^{-\frac{L^{2}}{2\left(\sigma_{0}^{2}+2 D t\right)}}\right]+\mathcal{O}\left(\Delta^{2}\right) \\
& =N_{0} \frac{\beta-\alpha}{2 \pi\left(\sigma_{0}^{2}+2 D t\right)} 2 L \Delta e^{-\frac{L^{2}}{2\left(\sigma_{0}^{2}+2 D t\right)}}+\mathcal{O}\left(\Delta^{2}\right) .
\end{aligned}
$$

Since the annular region has area,

$$
A=\frac{\beta-\alpha}{2 \pi} \pi\left[(L+\Delta)^{2}-(L-\Delta)^{2}\right]=2(\beta-\alpha) L \Delta
$$

we have

$$
N_{A}(t)=\frac{N_{0} A}{2 \pi\left(\sigma_{0}^{2}+2 D t\right)} e^{-\frac{L^{2}}{2\left(\sigma_{0}^{2}+2 D t\right)}}+\mathcal{O}\left(\Delta^{2}\right),
$$

giving the accuracy of (7).

\subsubsection{Sensitivity analysis}

Students soon realize that, in addition to their estimate of $D$, model validation requires measurements of $N_{0}, L$, and $\sigma_{0}$, each of which is likely to have some error attached. These errors are important to understand for both the students and for the instructors, since they give some notion of where to place experimental emphasis and what may cause divergence of results. A good exercise in multivariate calculus is to determine the relative sensitivity of (7) using the linear approximation

$$
\frac{\Delta N_{A}}{N_{A}} \approx \underbrace{\frac{D}{N_{A}} \frac{\partial N_{A}}{\partial D}}_{\epsilon_{D}} \frac{\Delta D}{D}+\underbrace{\frac{\sigma_{0}}{N_{A}} \frac{\partial N_{A}}{\partial \sigma_{0}}}_{\epsilon_{\sigma}} \frac{\Delta \sigma_{0}}{\sigma_{0}}+\underbrace{\frac{L}{N_{A}} \frac{\partial N_{A}}{\partial L}}_{\epsilon_{L}} \frac{\Delta L}{L}+\underbrace{\frac{N_{0}}{N_{A}} \frac{\partial N_{A}}{\partial N_{0}}}_{\epsilon_{N}} \frac{\Delta N_{0}}{N_{0}},
$$

where the sensitivity coefficients are

$$
\begin{aligned}
\epsilon_{D} & =-\frac{D t\left(-L^{2}+2 \sigma_{0}^{2}+4 D t\right)}{\left(\sigma_{0}^{2}+2 D t\right)^{2}}, \\
\epsilon_{\sigma} & =\frac{\sigma_{0}^{2}\left(L^{2}-2\left(\sigma_{0}^{2}+2 D t\right)\right)}{\left(\sigma_{0}^{2}+2 D t\right)^{2}}, \\
\epsilon_{L} & =-\frac{L^{2}}{\sigma_{0}^{2}+2 D t}, \quad \text { and } \quad \epsilon_{N}=1 .
\end{aligned}
$$

We suggest that students include such a sensitivity analysis in their lab reports along with an interpretation of the numerical values of these coefficients based on their experimentally determined parameters, as these results have implications for the design of the 
validation procedure. (Our results are reported in Section 3 and Figs. 9 and 10.) While many other mathematical extensions of this lab project are possible, we proceed now with the necessary biological background.

\subsection{Biological methods}

Although any small crustacean would serve for this exercise, those from the group of brine or fairy shrimp (family Anostraca) are readily available. We have successfully used Artemia franciscana (Crustacea, Branchiopoda) native to the Great Salt Lake (GSL), and the San Francisco Bay (USGS, 2009) (Fig. 1). Brine shrimp are collectively known as "Sea Monkeys". Below, we briefly describe their natural history and procedures for hatching and raising them to a size appropriate for this exercise. Additional information is available online as described in Appendix A.

\subsubsection{Natural history}

As their common name suggests, brine shrimp are adapted to high saline conditions and A. franciscana survives well in the GSL whose salinity ranges from 12-27\% (seawater is $3.5 \%$ ). Adults are approximately $10 \mathrm{~mm}$ long and feed primarily on GSL algae such as the flagellate Dunaliella viridis, but the species varies with season. Reproduction in $A$. franciscana is sexual with either live birth of first instar nauplii (i.e., first larval stage) or the production of cysts that are capable of prolonged diapause. The cysts are the "eggs" one buys in pet stores. Optimal reproduction in A. franciscana occurs at 14-17\% salinity, which in the GSL occurs in the south arm of the lake where persistent winds drive adults and floating cysts toward freshwater inflows and reduced salinity. The life span of a typical female is $6-9$ months and she can produce about 8 broods per year. Once born or hatched, 15-16 molts by nauplii are required for maturity. These life history details will depend on the particular local environmental conditions and the species. Additional life history information is available from resources listed in Appendix A.

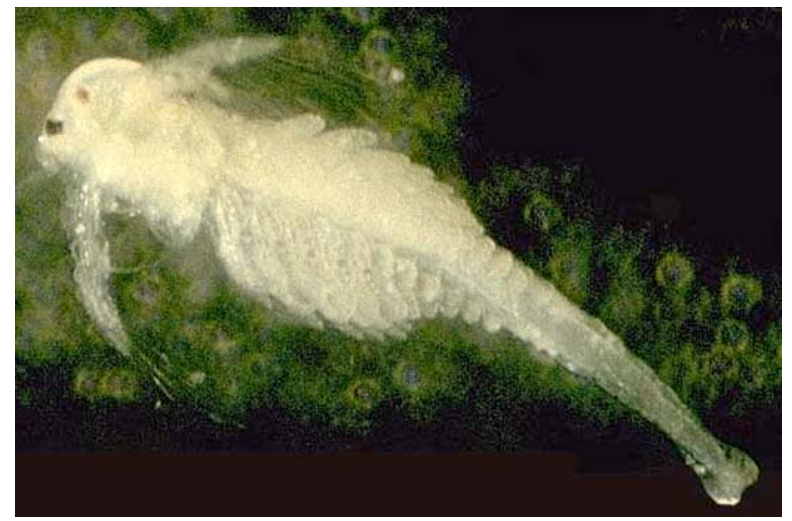

Fig. 1 Second instar nauplius of Artemia franciscana. The animal is about $1.5 \mathrm{~mm}$ in length. Photograph by Kevin Johnson was downloaded from http://ut.water.usgs.gov/shrimp/ accessed October 22, 2008. Used with permission. 


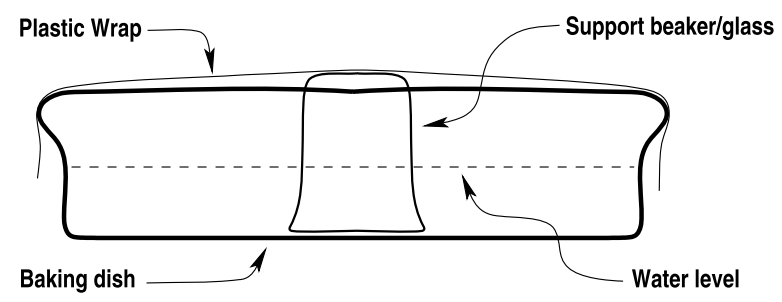

Fig. 2 Diagram of the baking dish set up used to hatch brine shrimp. The support beaker prevents the plastic wrap from collapsing on the saline water and shrimp.

\subsubsection{Hatching the shrimp from cysts}

The easiest method of obtaining the appropriate size class of brine shrimp nauplii for this laboratory exercise is to hatch the shrimp from commercially available cysts. Optimal hatching success of $A$. franciscana occurs in actual GSL water diluted to about $1 / 3$ of original salinity (c. 6-10\%). In the absence of the real thing, InstantOcean ${ }^{\circledR}$, at appropriate concentration, has a proper balance of ions. If neither is available, hatching will occur in a solution of $60 \mathrm{~g} /$ liter of table salt and tap water that has been exposed to the air for 3 days (to "de-gas" and remove fluoride and chlorine).

In a common household $9 \times 13$ inch $(23 \times 33 \mathrm{~cm})$ baking dish (preferably glass), add about 1 inch $(2.5 \mathrm{~cm})$ of salt water and pour the cysts onto the surface so that about $1 / 2$ to $1 / 3$ of the surface is covered with the brown cysts. Cover the dish with plastic kitchen wrap to prevent evaporation, using a small beaker or juice glass for support (Fig. 2). Early instar nauplii are delicate and undue turbulence can increase mortality so that aeration by atmospheric diffusion alone is best. Very gentle aeration with an aquarium bubbler pump and stone is possible, but avoid too much turbulence. Expect about 50\% hatching success. The dish can be left at room temperature in typical office ambient light intensities and cycles. No other incubation equipment is needed. Allow 3 days to obtain sufficient numbers for this exercise.

The experiment uses second nauplii shrimp which is the first feeding stage. A food source is not needed if the animals are used soon after hatching, but if you wish to keep them alive longer than a few days or attempt to grow them through additional nauplii stages, they are able to feed on bread yeast cells. Because brine shrimp adults are easily viewed with the unaided eye, have elaborate, beautiful anatomy, and interact with each other in small containers (especially males and females), the affective domain of learning (Table 1) can be addressed by providing students the opportunity to simply observe adults and nauplii in small containers. If dissecting microscopes or an overhead projector are available, this experience is greatly enhanced.

\subsubsection{Items needed for the labs}

The required materials for both lab procedures are:

1. A supply of second nauplius brine shrimp larvae (other members of the family Anostraca can be used).

2. Blunt, wide-aperture pipettes (plastic pipettes cut to an aperture of about $3-5 \mathrm{~mm}$ are best), 3 per group.

3. $150 \mathrm{~mm}$ by $15-20 \mathrm{~mm}$ deep Petri dishes, 2 per group (glass preferred or free of scratches if plastic). 

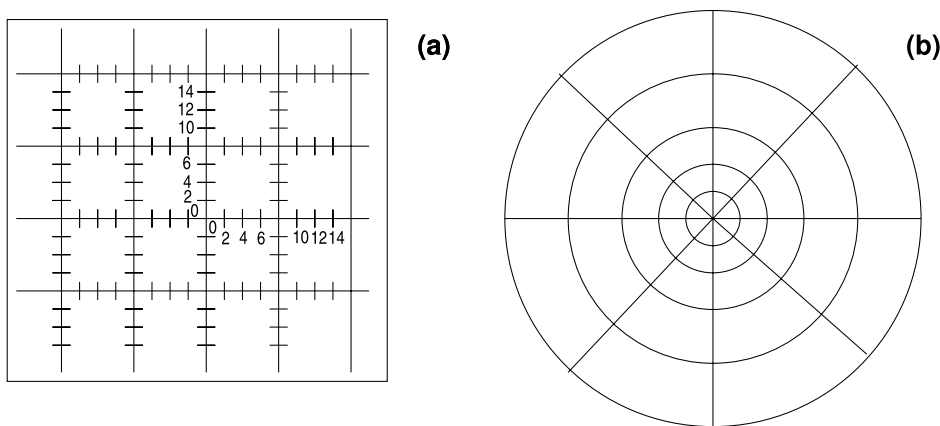

(b)

Fig. 3 Transparencies needed to measure shrimp movement (not actual size). (a) a grid for recording individual shrimp movement, (b) circles for measuring arrival densities. The URL for actual size templates is listed in Appendix A.

4. Overhead transparency gridded with $x-y$ coordinates (see Fig. 3(a)), 1 per group.

5. Overhead transparency marked in concentric circles (see Fig. 3(b)), 1 per group.

6. Small custard dishes or Petri dishes for transferring, 2 per group.

7. Overhead projector (ideally, 1 per group) or a light box or a multimedia projector that displays flat surfaces.

8. Stop watch or timer accurate to seconds, 1 per group.

9. (Optional) Video camera on a tripod to record movements.

10. (Optional) Adults for behavioral observation and student motivation.

Appendix A lists further references and websites with resources pertaining to this exercise.

\subsubsection{Individual movement procedure}

Before the exercise begins, the dish of second instar nauplii should be brought into the classroom and covered with foil, leaving one corner exposed. A bright light shined on the exposed corner will cause the shrimp to congregate so that high concentrations can be extracted for the arrival densities experiment. A dense cloud of organisms will form in 30-60 minutes.

To observe and record individual shrimp movement, the bottom of a Petri dish should be barely covered with salt water of the same salinity as that used to incubate the cysts. Place the gridded transparency (Fig. 3(a)) on the overhead projector and the dish on top of that centered on the origin of the grid. Use a pipette to deposit a small drop containing only a few shrimp. If neither a projector nor light box is available shine a small desk lamp obliquely on the Petri dish. Be aware that the shrimp are positively phototactic and will be attracted to the light.

After the shrimp are added to the Petri dish, one student times and records positions while the other calls out the $x$ and $y$ coordinates for an individual shrimp for as long as possible, at 5 or 10 second intervals (quicker is better but also more difficult; see Results below). When the shrimp nears the edge of the dish, observation should cease. This is repeated for 6-10 shrimp. The more shrimp and the longer the time traces, the better the estimates of $D$ (see Section 3.1 below). Best results seem to be obtained by following a shrimp starting at the center of the Petri dish for 60-90 seconds. Old style overhead 

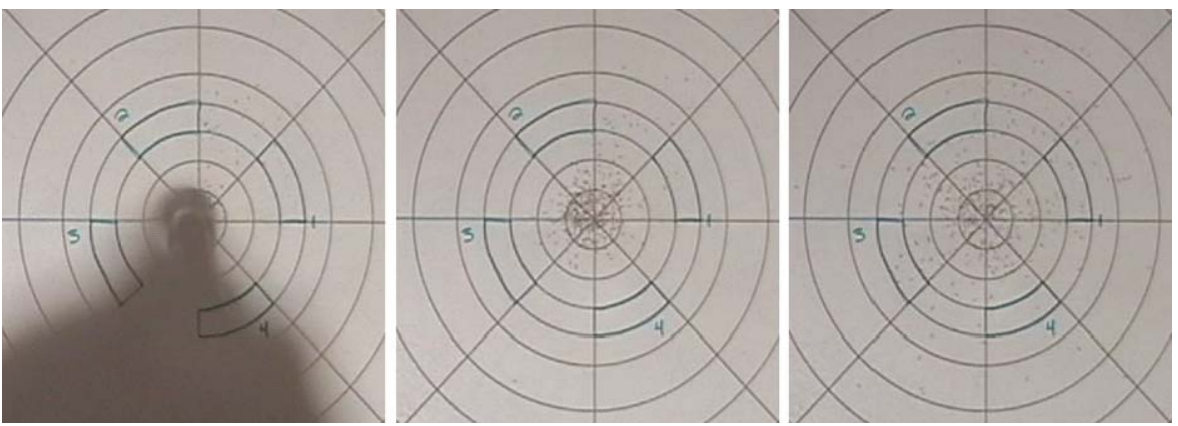

Fig. 4 Digital still images showing release and dispersal of shrimp within 10 seconds of the arrival densities procedure. The Petri dish image projected via overhead projector is approximately 5 feet in diameter. Individual shrimp, appearing as specks in these images, are easy to detect in part due to their movement.

projectors produce heat which will affect movement rates, so restarting the experiment with new water and shrimp every $5-8$ minutes is advisable. This is not a problem if you are using a video camera connected to a classroom digital projector.

\subsubsection{Arrival densities procedure}

As discussed above, this validation experiment simulates arrival in a spatial area after point release of invaders. Again, add salt water to barely cover a Petri dish; center the dish on top of the transparency with concentric rings (Fig. 3(b)) on the overhead projector. Use the pipette to extract a dense sample from the culture dish. Being careful to acquire as little water as possible, use the pipette to withdraw about 2-3 $\mathrm{ml}$ of shrimp culture.

Count accuracy is improved if an overhead projector is used to project the image on a wall. If a video camera is being used, start the camera. Carefully deposit the contents of the pipette in the center of the arena (Fig. 4). When releasing the shrimp, the pipette should be held vertically so as to minimize advective bias in the initial conditions. The student timer should note and record the area that the initial placement of shrimp occupies, by estimating the approximate radius of the population after release. This is an important parameter in the model ( $\sigma_{0}^{2}$ above).

We have found that a group of 5 students is optimal. One student times the intervals between counts ( 10 seconds) and records the values reported verbally by 4 student observers. Each student is assigned one region of the grid and reports the number of shrimp in the region when commanded by the timer. We repeat the observations at least twice using 4 regions equally near to the center of the grid and, in a separate experiment, 4 regions farther from the center. The near region should be outside the zone of the initial conditions, and the far region should not be too near the wall of the Petri dish. Students will want to design the experiment to last long enough to distinguish the projected pulse; in our experiments we continued for 2 to 3 minutes.

\subsection{Assessment strategies}

Due to the interactive nature of project-based learning, continual assessment of student learning is required to guide instructional strategies. For formative assessment, we listen carefully to student contributions during class discussions. Ideas offered by students are 
frequently incorporated into our presentation of new information; we ask many leading, open-ended questions to ensure that central ideas are eventually articulated by students and based on their individual experiences. During the class period, it is important for the instructors to circulate to the various groups, listen to questions raised, and problemsolving strategies. Generally, this is not passive; carefully framed questions and observations keep students engaged and facilitate their nascent ideas. We require a project from small groups containing a mix of biologists and mathematicians. We observe both intragroup learning (mainly math students teaching biologists) and intergroup learning, since we require on-going progress reports for the entire class. This presents students with alternative approaches to the same problem. When time permits, the whole class shares and discusses approaches to problems arising in data collection or model evaluation.

The target question, "How much like a random walk is the brine shrimp movement?" forces students to synthesize their understanding of the diffusion model in a written report. Individual or group reports are checked for mathematical accuracy to see that students have made true statements, correct calculations, and performed appropriate algorithms. Work is also evaluated for appropriate incorporation of mathematical equations and expressions as well as data and plots into a narrative that relays an acceptable understanding of the model and its applicability in the particular case of our experimental set-up. These latter aspects of mathematical writing are introduced not only in the diffusion lab; throughout the course, students prepare reports to hone their written communication talents.

\section{Results}

There are three flavors of results achieved using the brine shrimp diffusion experiment. First, there are estimates of diffusion constants and comparisons of arrival observations with predictions from both our students and ourselves. We present results both from our students' labs in Spring, 2008 as well as results obtained by the authors in Summer, 2008 to give potential instructors some feeling for what to expect from student results. Second, our analysis of the experiment is presented to help instructors evaluate, interpret, and anticipate student results, but not necessarily as a model of student results. There are procedural results to present as well; during our Summer experiments, we performed extensive videotaping of experiments so that we could ascertain the degree of error produced by human observation and also to provide a sufficiently extensive data set that bootstrapping would reveal the distribution of parameter results. The purpose of this is not to suggest that videotaping is a more effective way to collect data (although it is), but rather to reassure potential instructors that direct observation (which thoroughly engages students in a way that videotaped data does not) is "good enough." Third, there are the qualitative student responses to the lab, based on observations and reflections on what students have gained from this activity.

\subsection{Estimation of diffusion constant}

Figure 5 depicts observed mean squared displacements of 12 shrimp sampled at both 5 and 10 second intervals. Several different slopes can be determined depending on the length of the data set considered. This is a good example of the real world of messy data 

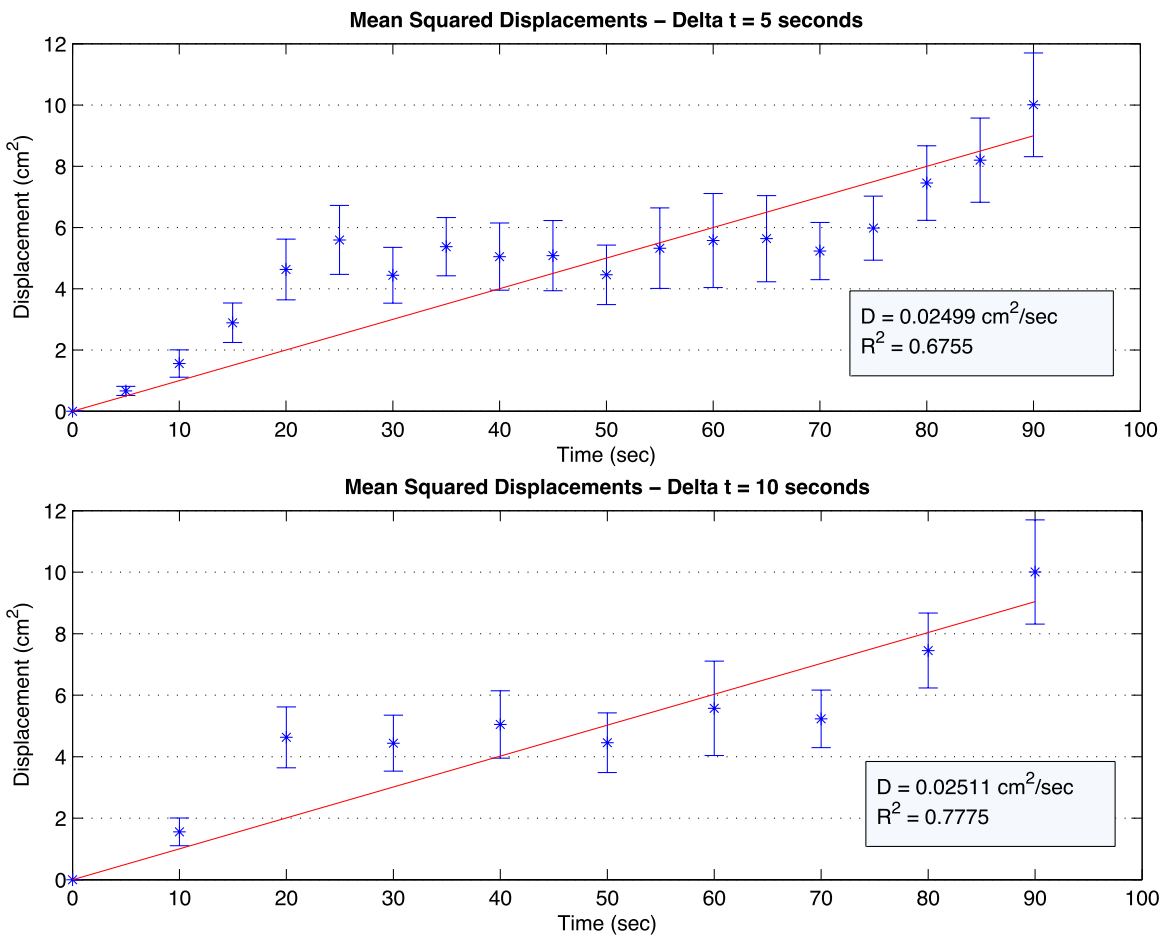

Fig. 5 The mean squared displacements for shrimp movement are shown above when data were collected at 5 and 10 second intervals. Each point represents the average of roughly 12 shrimp.

that students must confront. To determine how often the position of each shrimp should be recorded during the individual movement procedure, Fig. 5 shows the effects of collecting data every 5 seconds and every 10 seconds on the estimate of the diffusion constant, $D$. We conclude that the time interval used does not have a large effect on the estimate of $D$, and the appropriate time interval should be determined based upon the ability of the students conducting the data collection. In both our and our students' experience, the use of 5-second intervals is about the limit sustainable for calling out and recording positions, but excitement is maintained; the use of 10 -second intervals is very easy to keep up with, but the time spacing begins to feel tedious because not enough is happening.

A second experimental design constraint is the length of time that students should follow an individual shrimp. As mentioned in Section 2.2.4, observation of an individual shrimp should cease when it comes in contact with the edge, as its movement can no longer be considered random. However, each shrimp will hit the edge after a different length of time, and accordingly longer data tracks will begin sampling only slower shrimp. Figure 6 shows the effect of the total length of data collection on the estimate of $D$. We conclude that 80 to 90 seconds is an appropriate length of time to record the positions of shrimp that do not contact the edge before that point. In taking a large number of data samples, we have found that approximately $75 \%$ of the data tracks for individual shrimp 
Table 2 Results of bootstrapping the data collected from the students in the class, and from our own experiment in real time and the video recording. Samples of size $N$ were randomly selected from the total tracks collected. The mean diffusion coefficient $D$ and standard deviation (in $\mathrm{cm}^{2} / \mathrm{s}$ ) were computed from repeated randomly selected samples

\begin{tabular}{|c|c|c|c|c|c|c|}
\hline \multicolumn{7}{|c|}{ For video data: } \\
\hline $\bar{N}$ & 10 & 20 & 30 & 40 & & all 43 \\
\hline mean $D$ & 0.0250 & 0.0250 & 0.0250 & 0.0250 & & 0.0250 \\
\hline SD $D$ & 0.00721 & 0.00499 & 0.00410 & 0.00348 & & \\
\hline \multicolumn{7}{|c|}{ For class data: } \\
\hline$N$ & 15 & 20 & 30 & 40 & 50 & all 52 \\
\hline mean $D$ & 0.0413 & 0.0414 & 0.0412 & 0.0412 & 0.0414 & 0.0413 \\
\hline $\operatorname{SD} D$ & 0.0113 & 0.00995 & 0.00794 & 0.00693 & 0.00623 & \\
\hline
\end{tabular}

For the original real time data we collected:

\begin{tabular}{lllr}
\hline$N$ & 10 & 15 & all 19 \\
\hline mean $D$ & 0.0298 & 0.0299 & 0.0299 \\
SD $D$ & 0.0101 & 0.00830 & \\
\hline
\end{tabular}

For the class data and the data we collected:

\begin{tabular}{lllllll}
\hline$N$ & 10 & 20 & 30 & 40 & 50 & all 71 \\
\hline mean $D$ & 0.0378 & 0.0379 & 0.0378 & 0.0377 & 0.0378 & 0.0378 \\
SD $D$ & 0.0113 & 0.00934 & 0.00767 & 0.00655 & 0.00592 & \\
\hline
\end{tabular}

displacement will contain information for up to 90 seconds and results seem fairly stable up to that time.

The last experimental design consideration is the number of data tracks, $N$, required to produce reasonable results. Through bootstrapping both the real-time data and the video data, we obtained the results shown in Table 2. Results vary between class and author data due to variance in experimental conditions; experiments were performed in different rooms, at differing temperatures and light conditions, with shrimp of different broods, leading to diffusion constants almost twice as large. With only 10 observations, the standard deviation of estimates hovers around $28 \%$ and decreases inversely with $\sqrt{N}$. Hence, at 40 observations, the standard deviation is approximately $14 \%$ of the estimate of $D$. Statisticians would no doubt tell us that $N$ should be much larger; on the other hand, to get the standard deviation down to $10 \%$ would require on the order of 80 observations $\left(28^{2} / 10^{2} \approx 7.8\right)$. As discussed below, sensitivity of the validation experiment to $D$ is relatively low. Since 10 observations require 15-20 minutes, a reasonable goal given sufficient class time, would be 20 observations with an anticipated error of $20 \%$ in estimates of $D$.

\subsection{Arrival densities procedure}

Both students and the authors observed arrival numbers in two counting areas (near and far, $L=1.83 \mathrm{~cm}$ and $3.65 \mathrm{~cm}$, respectively). The goal of the experiment is to determine whether or not brine shrimp are following a direct prediction of the diffusion model. 


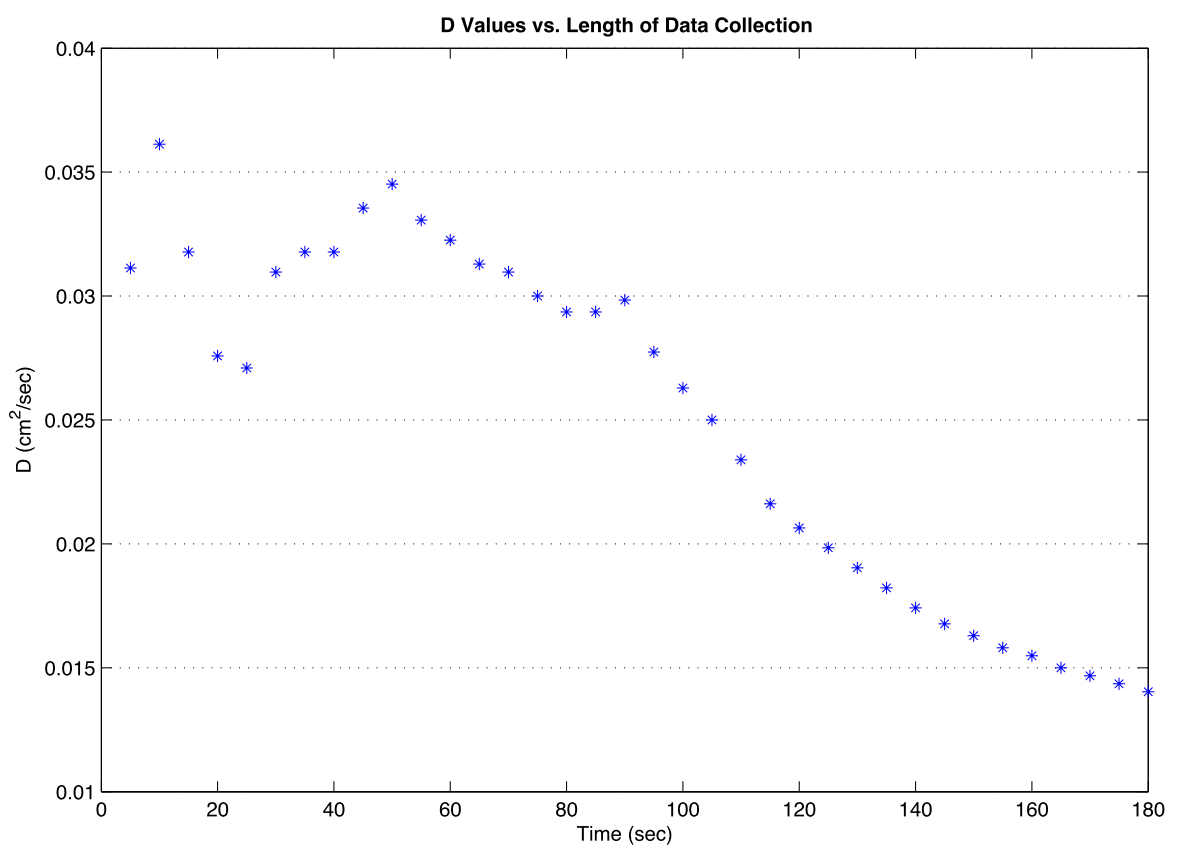

Fig. 6 The estimated diffusion coefficients are pictured as a function of the duration of data collection. The points are computed from approximately 12 shrimp in the first 100 seconds, but data became unavailable as shrimp hit the edges of the dish so points after 100 seconds arise from averages of 11 down to 3 shrimp.

Students immediately realize that $D$ is not the only parameter required; while other parameters come from direct measurement, the total number of shrimp released, $N_{0}$, must be determined directly by counting. We compared direct counts with stop-action counts of video data to ascertain the error in human counting. Using our real-time data, $N_{0}$ was calculated with a $3 \%$ error and $15 \%$ error in the two separate trials when compared to exact counts from the video recordings. Typically, real-time numbers were lower than numbers found when using the videotaped data, but since the sensitivity of the predictions to error in $N_{0}$ is direct, these errors are relatively trivial from the standpoint of validation/falsification.

Figure 7 shows the arrival of shrimp at two different regions for both student real-time data and numbers gathered from a video recording of the same trials. This figure indicates that counting at arrival areas is unlikely to be the source of variability; the arrival process itself is inherently stochastic and counting error seems to contribute little to overall variability. Figure 8 shows observed shrimp arrivals with the predicted arrivals based on the diffusion model. The figure shows that variability in counts at the arrival area is obviously high, of the same order as the expected number. Most students, in fact, looking at the raw data, are convinced that the diffusion model is a failure and that there is no clear pulse of arrival. However, when they plot model predictions their perceptions reorganize. As often happens, inflicting a predicted curve on observational data brings out elements of the global behavior which might otherwise be missed. The variability makes it difficult to definitively validate the diffusion model (particularly given its sensitivity to 

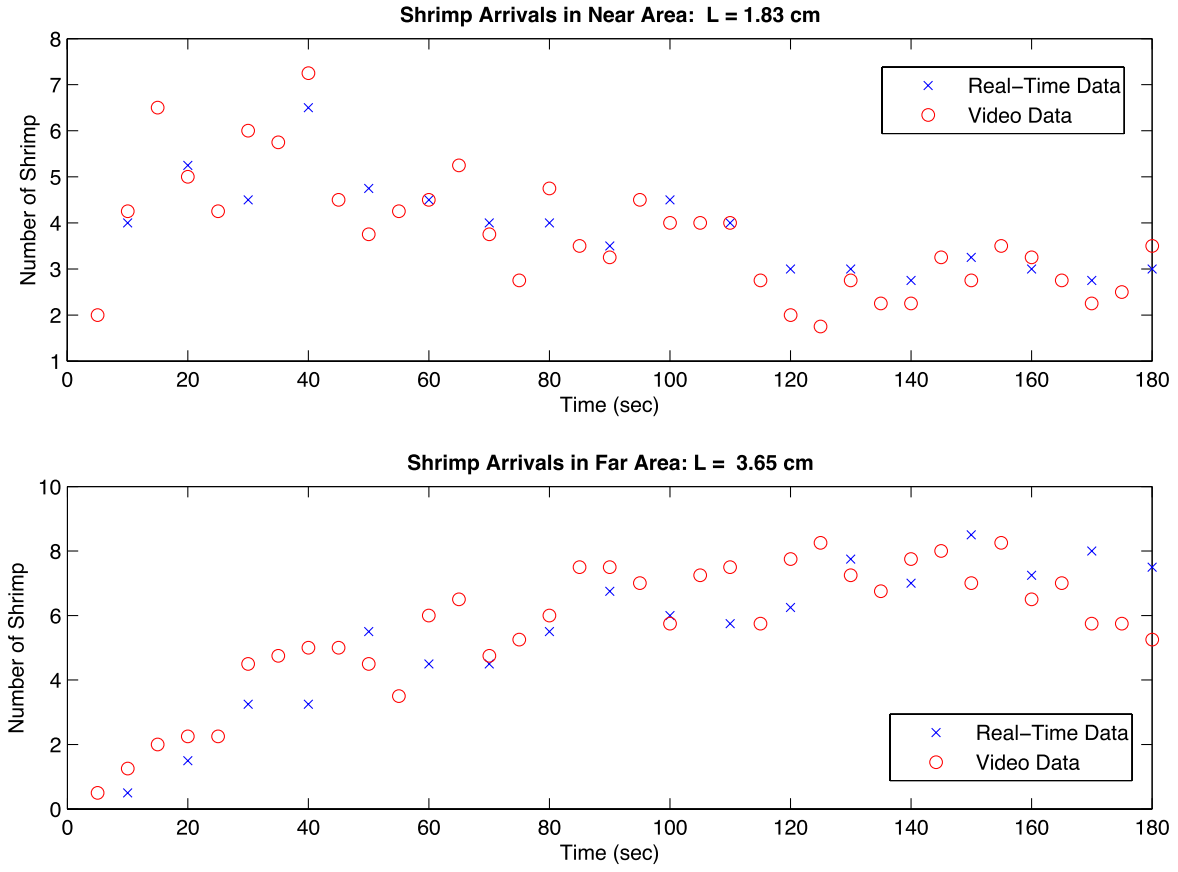

Fig. 7 Pictured above is the number of shrimp counted in each region from our visual inspection in the arrival densities procedure for near (top) and far (bottom) areas. We compare this to the more careful method of counting shrimp in still images of the video recorded data.
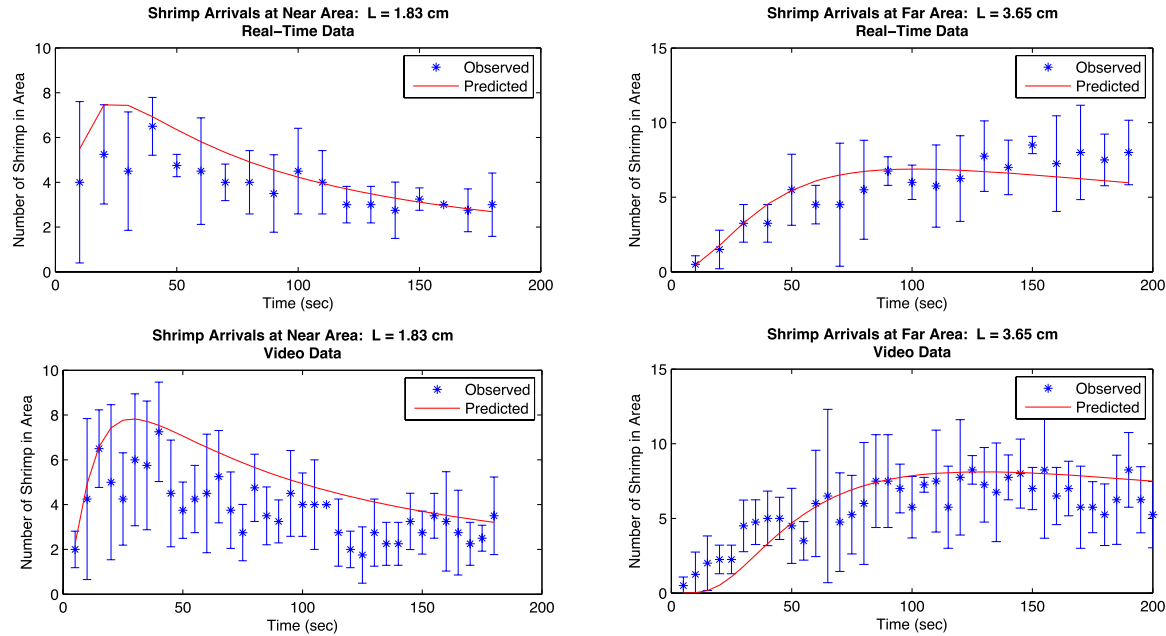

Fig. 8 Predicted and observed arrival densities at near (top panels) and far (bottom panels) regions for real time (left panels) and video taped (right panels) data. Symbols are data, solid line are prediction from Eq. (7). Error bars are \pm 1 standard deviation. 
the measurements, see below), but the model's ability to organize the overall behavior of the observations makes it difficult to falsify as well.

\subsection{Parameter sensitivities}

To assess elements of the experimental protocol, we performed a sensitivity analysis on selected parameters. For nominal measurements of $L=1.4 \mathrm{~cm}, \sigma_{0}=0.5 \mathrm{~cm}, D=2.6 \times$ $10^{-2} \mathrm{~cm}^{2} / \mathrm{s}$, the peak of the arrival pulse occurs at $t \approx 134.3 \mathrm{~s}$, at which time, from Eqs. (9-11), the elasticities are

$$
\epsilon_{D}=0.11, \quad \epsilon_{\sigma}=0.01, \quad \epsilon_{L}=-2.23 .
$$

Recalling that $\epsilon_{N}=1$ (Eq. (11)), we see that accuracy at the pulse is more than twice as sensitive to measurement of $L$ as measurements of $N_{0}$. A $5 \%$ error in estimating the population size will create a $5 \%$ error in measurements of population at the peak of the pulse; a $5 \%$ error in estimating $L$ produces an $11 \%$ error. Measurements at the pulse will be relatively insensitive to errors in either $D$ or $\sigma$. Earlier (e.g., $t=15$ )

$$
\epsilon_{D}=4.17, \quad \epsilon_{\sigma}=3.18, \quad \epsilon_{L}=-13.5 .
$$

While only a small fraction of the population will have arrived at this time, this indicates that measurements of the initial ramp of the pulse may be very sensitive to parametric error, particularly in measurements of $L$. Figures 9 and 10 show the sensitivity of the model to changes in the parameters over the entire length of data collection. We discuss these kinds of results in class in the context of experimental design. For example, "If we were to repeat the experiment, how would you change the observational techniques?" Or,

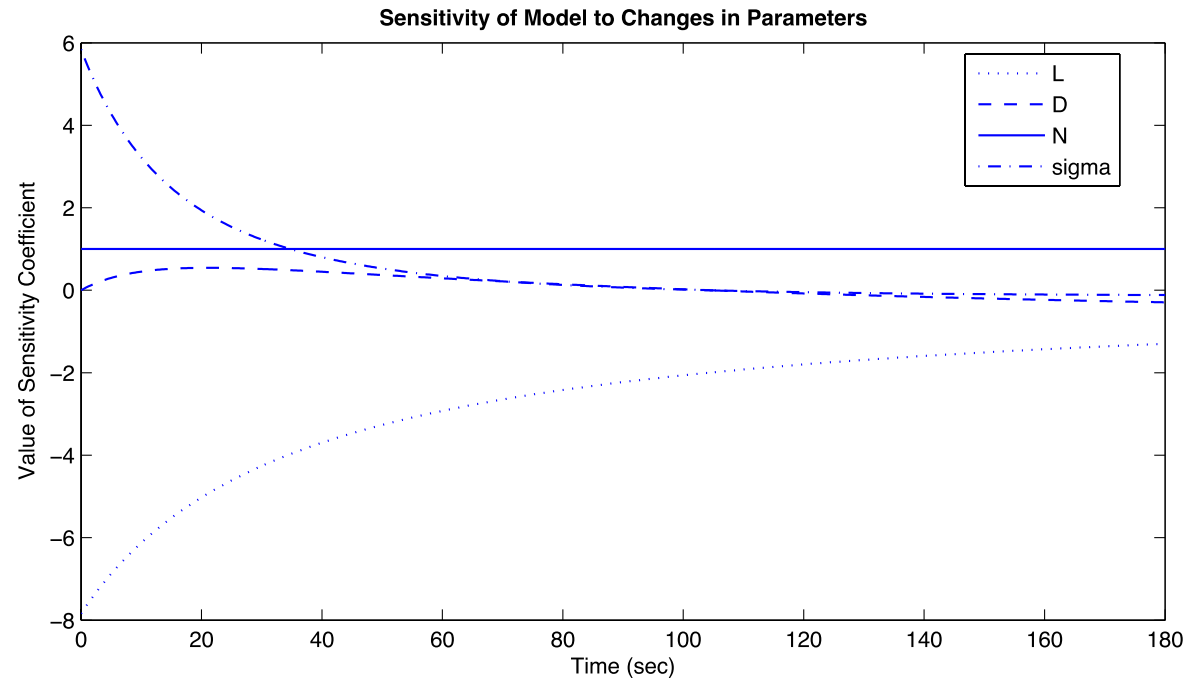

Fig. 9 The parameter sensitivity coefficients from Eqs. (9-11) are shown. The parameter values for $L=1.4 \mathrm{~cm}, \sigma_{0}=0.5 \mathrm{~cm}, D=2.6 \times 10^{-2} \mathrm{~cm}^{2} / \mathrm{s}$ are used as a base measures in the expressions for each coefficient. 

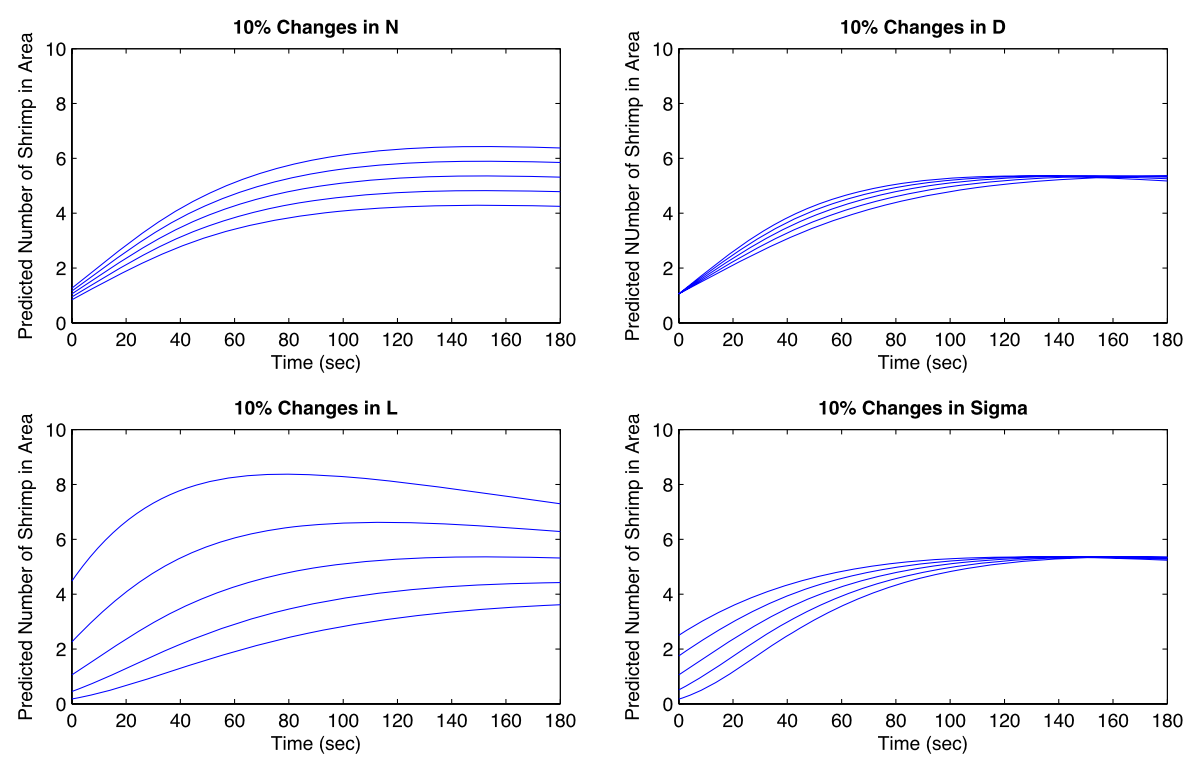

Fig. 10 The predicted arrivals from Eq. (7) are plotted here for 10\% variations in each parameter to show sensitivity to parameters.

these discussions can profitably occur before the students collect data. For the instructor's benefit, designing an arrival area with $L=2 \mathrm{~cm}$ is probably optimal for balancing the desire for a clear peak relatively, but not too early (as opposed to the broad, flat peak illustrated on the right-hand side of Fig. 8) and low sensitivity to measurement errors.

\subsection{Evaluation of student learning}

Ultimately, the success of the lab lies in its value as a pedagogical experience for students. Our evaluation of what students have learned from this activity comes from our observations during the course, student reports on the project, and emailed reflection questionnaires received from the course instructors and recent students. According to the instructors' documentation, student progress was clearly evident from initial discussions in class, the tenor of group discussions during experiments, questions posed during office hours, and the final lab write-up, which was generally quite professional. From this, we are convinced students achieve a wide array of learning objectives, some of which are tabulated in Column 3 of Table 1 . Students not only gain a basic understanding of the model and topic, but they display a diversity of cognitive engagement and performanceexhibiting remarkable achievement of discovery, application, creative thinking, appreciation, and willingness-to-try learning objectives. These types of objectives are rarely reached through lecture alone.

To illustrate what students acquire through this exercise, we include a few excerpts from their reports. Showing a general understanding of random walks, one student writes, "In a two-dimensional world, there are only two ways that the brine shrimp or any other organism can move. After they make a move they may reorient and make another movement at random." Students make graphs displaying mean squared distance as a function 
of time and display their linear regression results (similar to Fig. 5). One report states, "The resulting averages of distance moved from the origin show shrimp disperse from the origin at a dependable rate. The slope of the trend line allows us to state the predicted diffusion constant for shrimp." Some students report a great deal of detail about their validation experimental design and include tables of raw data as well as graphs to illustrate their conclusions, while others more briefly summarize methodology and incorporate graphs of their recorded data compared to model predictions (similar to Fig. 8). Two main deficiencies that continually arise in student reports during this course are: (1) intelligent and well-formed writing that surrounds the equations, and (2) figure and table captions. Instructors were initially surprised at the number of students who either do not read or do not conform to writing standards in journal articles. To teach writing skills, they collect preliminary versions of reports and give detailed feedback before the final product is due.

Whether or not students' results align squarely with the diffusion model, they demonstrate their analytical thinking through the process and interpretation. Realizing the importance of precise experimental technique and accuracy of calculations, one student reports, "It is possible that errors were made during the experiments or in the calculation of the diffusion constant that made for the incongruity. It is also possible (although I checked and rechecked it) that the diffusion equation predictions were not computed correctly."

The project naturally leads students to raise mathematically and scientifically important issues. For example, students discussed the effect of a no-flux boundary (the edge of the Petri dish) and raised concern about trying to balance where "removing outliers" becomes "misrepresenting data." One student included a paired T-test, with the pairs of the test being the times at the observed and predicted values, and concluded that the model and data are significantly different from one another. To explain this discrepancy, she wrote, "The diffusion equation may not have worked to predict the movement of brine shrimp because the shrimp were moving around in circles. Maybe if we would have collected data for areas closer to the center we would have gotten better results."

Students notice that at small scales the shrimp movement is clearly correlated with past direction and speed, but on larger scales where they are pointing and where they are going seems pretty random compared to what they were doing 5-10 minutes prior. Hence, students think a lot about whether the basic premise of the model is satisfied or not, and what not satisfying the premise might mean. Another student concludes, "Shrimp do follow the diffusion model, but are prone to exhibiting a more organic unpredictability of location. They seem to find a comfortable distance of dispersion and then stagnate to forage."

The challenges and rewards of using personally collected data from living creatures helps students gain a deeper appreciation for the process of fitting and evaluating a mathematical model. Students reported they enjoyed learning through the hands-on labs and collaboration with peers and instructors. They also gained confidence, initially bewildered by the notion of describing population dispersal mathematically, then amazed they can work with and understand a PDE. In the emailed reflection, one student wrote, "It was the first time I had used collected data to parameterize a PDE model. I learned the difficulty of adding a spatial element to a mathematical model." Particularly, the math students expressed appreciation for the challenges of working with living organisms. "I learned that it is hard to keep data collection consistent and accurate, especially when observing something that moves quickly and unpredictably like brine shrimp." It is not surprising that a large number of students who have taken this course have pursued research projects outside of class in biomathematics. 


\section{Discussion}

The brine shrimp lab is an example of a project-based learning experience for students that kindles interest in advanced mathematics and provides a vehicle for friendly collaboration among math, biology and math education faculty. We provide a summary of the main teaching components and brief recommendations for implementation in Table 3. Our experience confirms that in addition to being a tractable lab (without requiring sophisticated equipment), the project is also mathematically reasonable as a vehicle for studying the classical diffusion model, and consistent with reform efforts in improving mathematics instruction. The biological preparation is relatively simple, making it feasible to incorporate into classes in PDEs, vector calculus, mathematical modeling, advanced statistics classes, and stochastic processes. Each of these specific classes would emphasize different aspects of the exercise as appropriate to the subject matter. For example, a statistics class might emphasize goodness-of-fit tests to a Gaussian distribution, a PDE class might develop error terms to approximations to flux rates, or a class on stochastic processes

Table 3 Summary of the instructional procedures and our recommendations for implementation

\section{Instructional Procedures}

1. Derivation of the model Introduce the derivation and solution of the diffusion equation as a 2-dimensional random walk through lecture and discussion. Lead students to the question of the project, "Is diffusion a good model for brine shrimp movement?" and begin a discussion of appropriate experiments

2. Individual Movement Lab Allow students to get comfortable with the equipment and handling of the shrimp, also allow some flexibility in the way they choose to collect data keeping the goals of the procedure in mind

3. Regression Lecture on the mathematical framework for fitting a line to the data gathered during the individual movement lab. Introduce and review required MATLAB commands as students find their diffusion coefficients. Discuss advantages of pooling the data and provide a way for groups to share results

\section{Model Validation Through discussion lead} students through the qualitative behavior expected in the model for the arrival density experiment. Also, present the mathematical formulation. Conduct the arrival density lab to collect data and estimate parameters

\section{Follow up discussion and assessment Follow} up with a discussion of findings and clarify the assigned report. Discuss the optional parameter sensitivity analysis and other issues the experiments raised to be addressed in the written reports

\section{Recommendations}

Emphasize the construct-a-concept learning level as students formulate an understanding of the diffusion model. Also, be in tune with the level of mathematical precision students use in their communication. Throughout the exercise, guide them toward using more mathematical language

Emphasize discover-a-relationship level learning. Encourage students to make connections and raise issues regarding the experimental procedure and mathematics

Students practice applying the algorithms on their own and check results with one another

Allow students to deductively reason through the qualitative behavior and mathematics. Emphasize the application learning level

Promote and reward divergent thinking. Follow through on suggestions and ideas students raise through their experience 
might devote more attention to the interface of individual probabilistic events and average stochastics of ensemble behavior.

A key component to the instructional effectiveness of this project is the classroom environment that fosters learning because of the communication and interaction style among the professors and students. The fact that the course instructors consist of a genuine interdisciplinary team, one from biology and one from mathematics, with a long productive history as research collaborators, certainly provides students with an exemplary model. Students might learn how to be a part of effective teams simply by observing the interaction style and cooperation between the instructors. However, this aspect of our implementation of the project is not a necessity. Another skillful instructor in tune with the working styles and personalities of students in class can orchestrate an environment conducive to effective teamwork. The instructors of the course employ a variety of strategies for creating a community of inquiry in the discussions (compare with Goos, 2004), and predominantly these strategies are realized through the communication style in the class. By using descriptive language, and avoiding the typical Initiate-Response-Evaluate cycles that dominate traditional classroom communication, the instructors create an environment in which students no longer fear but embrace failure and are invested in gathering and interpreting experimental results (Cangelosi, 2003). The inherent uncertainty of a biological experiment puts students and faculty on equal footing for discussion as lectures cannot. Hence, classroom discourse is enhanced since conversations arise addressing the variability in the methodology and findings. Students engage in reasoning-level (rather than memory-level) question-and-answer sessions throughout the project. Ideas offered by students are valued, frequently followed, and not judged by the instructor.

Another essential component to the success of this project is the direct experience of gathering data from living organisms. Presenting students with prepared, published, or hypothetical data fails to engage them in scientific inquiry. Exploring computer simulations of random walks can help involve students in conjecturing and inductive reasoning, yet the simulation approach still greatly diminishes the investment and connection students feel when working with real brine shrimp. Possibly, this is because we are deeply wired to try to understand the motion of other agents in the real world, which "hooks" students. The time and energy necessary to gather data gives students ownership and motivation. The novelty of handling living things in a mathematical context inspires curiosity leading to the creative examination of the relationship between diffusion solutions and observational reality. Mathematics changes its meaning from an abstract logical game to a sense-making language adding predictability to erratic situations.

There is a trade-off between the didactic and discovery nature of this project. The discovery process takes time, is messy, inexact, and likely to be uncomfortable territory for a didactic researcher/professor used to making polished presentations of carefully edited essential information and choice examples. Especially at the start of a new unit of instruction, the initial biological question is vague: "Do guppies forage optimally? Do shrimp move randomly?" These types of questions usually elicit long periods of silence. One has to be careful that expert input is held until the class has had a chance to struggle. Less information and mathematical knowledge will be delivered, but discovery-based teaching involves students more directly in the activities of our profession. Our hope is that readers will try this or similar exercises in their classes, enjoy the incorporation of project-based learning in their own teaching, and that our efforts will save time in class preparation. 


\section{Appendix A: Resources}

An excellent source for many of the items needed is either a colleague from the local biology department or:

Carolina Biological Supply Company

2700 York Road

Burlington, NC 27215

www.carolina.com

voice: $800-334-5551$

The item references below are from CBSC Catalog 77, 2008.

1. $150 \mathrm{~mm}$ Petri dishes: local chemistry stores or CBSC (glass: item FA-74-1164, plastic: FA-74-1254)

2. brine shrimp: local pet store or CBSC: hatching kit (everything needed in one package: eggs, pump, salt: FA-14-2214); eggs only: FA-14-2240; adults for optional behavioral observation: FA-14-2230)

3. Large plastic pipettes: CBSC: FA-73-6988

4. Information on the GSL and brine shrimps:

http://ut.water.usgs.gov/shrimp/index.html http://wildlife.utah.gov/gsl/brineshrimp/index.php

http://www.aquaculture.ugent.be/index.htm

5. PDF files for the templates can be downloaded from:

http://www.math.usu.edu/ kohler

6. A Java simulation of Artemia movement, growth, and reproduction:

http://www.captain.at/artemia-simulation.php

7. Webpage for "Applied Mathematics in Biology" USU Biol/Math 4230:

http://cc.usu.edu/ jhaefner/AMBcourse.html

8. Additional laboratory exercises for biomathematics:

http://cc.usu.edu/ jhaefner/BioMathLab.html

\section{References}

Berg, H.C., 1993. Random Walks in Biology. Princeton University Press, Princeton.

Cangelosi, J.S., 2003. Teaching Mathematics in Secondary and Middle School: An Interactive Approach, 3rd edn. Merrill/Prentice Hall, Columbus/New York.

Erickson, D.K., 1999. A problem-based approach to mathematics education. Math. Teach. 92(6), 516-521. 
Fisher, R., 1937. The wave of advance of advantageous genes. Ann. Eugen. 7, 335-369.

Goos, M., 2004. Learning mathematics in a community of inquiry. J. Res. Math. Educ. 35(4), 258-291.

Hmelo-Silver, C.E., 2004. Problem-based learning: what and how do students learn? Educ. Psychol. Rev. 16(3), 235-266.

Krajcik, J.S., Blumenfeld, P.C., Marx, R.W., Soloway, E., 1994. A collaborative model for helping middle grade science teachers learn project-based instruction. Elem. Sch. J. 94(5), 483-497.

Krantz, S.G., 1999. How to Teach Mathematics, 2nd edn. American Mathematical Society, Providence.

Lay, D.C., 2003. Linear Algebra and Its Applications, 3rd edn. Pearson Education, Upper Saddle River.

Ledder, G. (ed.), 2008. PRIMUS Problems, Resources, and Issues in Mathematics Undergraduate Studies, vol. 18. Taylor \& Francis, London.

Miller, L.N. (ed.), 1994. Ecology, vol. 75. Ecological Society of America, Ithaca.

Pollatsek, H., Barker, W., Bressoud, D., Epp, S., Ganter, S., Haver, B., 2004. Undergraduate Programs and Courses in the Mathematical Sciences: CUPM Curriculum Guide 2004. The Mathematical Association of America, Washington.

Powell, J.A., Cangelosi, J.S., Harris, A.M., 1998. Games to teach mathematical modeling. SIAM Rev. 40, 87-95.

Rassmussen, C., Marrongelle, K., 2006. Pedagogical content tools: integrating student reasoning and mathematics in instruction. J. Res. Math. Educ. 37(5), 388-420.

Rogers, E.C., Reynolds, B.E., Davidson, N.A., Thomas, A.D. (eds.), 2001. Cooperative Learning in Undergraduate Mathematics: Issues that Matter \& Strategies that Work. The Mathematical Association of America, Washington.

Skellam, J., 1951. Random dispersal in theoretical populations. Biometrika 38, 196-218.

Solomon, G., 2003. Project-based learning: A primer. Technol. Learn. 23(6), 20-30.

Steen, L.A. (ed.), 2005. Math \& Bio 2010: Linking Undergraduate Disciplines. The Mathematical Association of America, Washington.

Thomas, J.W., 2000. A Review of Research on Project-Based Learning. The Autodesk Foundation, San Rafael.

Turchin, P., 1998. Quantitative Analysis of Movement: Measuring and Modeling Population Redistribution in Animals and Plants. Sinauer, Sunderland.

Turing, A., 1952. The chemical basis of morphogenesis. Philos. Trans. R. Soc. Lond. B 237(641), 37-72.

USGS, 2009, Brine shrimp and ecology of great salt lake. http://ut.water.usgs.gov/greatsaltlake/shrimp/. Accessed 25 March 2009. 